\title{
Nutrition Indicators, Physical Function, and Health-Related Quality of Life in Breast Cancer Patients
}

\author{
Krystal Ng Lu Shin ${ }^{1,2}$, Chan Yoke Mun ${ }^{1,3 *}$, Zalilah Mohd Shariff ${ }^{1}$
}

\begin{abstract}
Objective: This study aimed to investigate how nutrition indicators and physical function may influence Health-related Quality of Life (HRQoL) of breast cancer patients undergoing treatment. Methods: This was a cross sectional study among a total of 163 breast cancer patients. Series of measurements including anthropometry, biochemical, and dietary were employed to assess patients' nutritional status while physical function was assessed by handgrip strength. HRQoL of patients was determined using European Organization for Research and Treatment of Cancer quality of life questionnaire Core 30 (EORTC-QLQ-C30) version 3.0. Multiple linear regression was used to identify factors associated with HRQoL. Results: Breast cancer patients perceived moderately their overall quality of life (QoL), with the mean global health status (GHS) score of 69.12. Emotional functioning was the poorest functional scale while fatigue was the most distressing symptom presented by the patients. Approximately $20 \%$ of patients had low corrected arm muscle area while more than half had low hemoglobin level. More than $90 \%$ of patients did not meet the overall dietary recommendation and had poor handgrip strength. Mid-upper arm circumference (MUAC) was associated with GHS ( $\beta$ : $0.906 ; 95 \%$ CI: $0.22,1.56)$ and cognitive functioning $(\beta:-1.543 ; 95 \%$ CI: $-3.07,-0.01)$. Handgrip strength was positively associated with most of HRQoL outcomes. Conclusions: Breast cancer patients reported overall good nutritional status and moderate QoL during treatment. Being well-nourished improved HRQoL and handgrip strength could be a potential proxy for functional outcomes as well as overall QoL.
\end{abstract}

Keywords: Breast cancer- Handgrip strength- health-related quality of life- nutritional status

Asian Pac J Cancer Prev, 21 (7), 1939-1950

\section{Introduction}

A new paradigm for cancer care has been intensively deliberated, evolving from an aggressive cancer cure treatment to a life-prolonging treatment with the main consideration of Health-related quality of life (HRQoL) (Kelley and Meier, 2010; Patel et al., 2014). While HRQoL is gaining recognition as a prognostic indicator of cancer survival (Quinten et al., 2009) and has been equally acknowledged as an important clinical endpoint in cancer research (Fiteni et al., 2014), HRQoL outcomes should be emphasized in all cancer patients regardless of disease stages. It is essential for healthcare professionals to understand one's perception of health or life status throughout cancer treatment as this could imply an effective clinical decision making, pertaining to better treatment adherence (Kane et al., 2014; Kelley and Meier, 2010). With improved management of functional disabilities or HRQoL impairment, a lower burden of hospitalization for cancer patients is anticipated.

Breast cancer population had received considerable attention in light of its high morbidity and mortality rates (Ferlay et al., 2014). Earlier detection awareness and advances in cancer treatment have greatly improved breast cancer survival rates, particularly in high-income countries (Torre et al., 2015). Nonetheless, treatment-related side effects on nutritional status such as nausea and vomiting, appetite loss and diarrhea, which negatively affect patients' functional status and HRQoL (Dang et al., 2016) are commonly reported. While mounting evidence shows malnutrition is associated with decreasing HRQoL in cancer patients (Lis et al., 2012; Yu et al., 2013), the relationship between nutritional status and HRQoL is under-studied for breast cancer patients, who are at lower risk of under-nutrition as compared to other cancers (Lis et al., 2012). In spite of the negative impact of altered taste perception on calorie intake (Boltong et al., 2014; de Vries et al., 2017), weight gain was increasingly reported for breast cancer patients even during treatment (van den Berg et al., 2017), which may decrease their physical function and overall quality of life (QoL) (Fang et al., 2013), suggesting healthy eating habit for general

${ }^{1}$ Department of Nutrition and Dietetics, Faculty of Medicine and Health Sciences, Universiti Putra Malaysia, Malaysia. ${ }^{2}$ Department of Health Education, Literacy, Promotion and Policy, National Cancer Society of Malaysia, Malaysia. ${ }^{3}$ Research Centre of Excellence Nutrition and Non-communicable Diseases, Faculty of Medicine and Health Sciences, Universiti Putra Malaysia, Malaysia.*For Correspondence: yokemun_chan@yahoo.com 
health maintenance among the breast cancer patients is critical. Acknowledging nutrition disorders are modifiable determinants of health outcomes especially for impaired HRQoL, an effort to explore on the nutrition-related factors and cancer HRQoL is indispensable. On the other hand, primary treatment of axillary lymph node dissection can lead to muscle dysfunction and reduced limitation of doing routine activities (Hidding et al., 2014), elucidating the long term impairment of physical function and HRQoL. Investigating the association between muscle weakness and various aspects of functional health would enable a better understanding of the implication of physical limitation in overall QoL.

Studies on HRQoL have been focusing among survivors, while the exploration of HRQoL determinants especially for breast cancer patients who receiving treatment is relatively scarce. This finding allows us to formulate more effective strategies to improve patients' HRQoL at this critical period, which could assist the healthcare providers to make clinical decision wisely. This study utilizes the objective measure of handgrip strength to evaluate physical function, in order to minimize the self-report bias in HRQoL questionnaire. The parameters of anthropometric, biochemical, and diet quality were assessed to provide valuable information regarding the association of nutritional factors with HRQoL. Specifically, this study aimed to determine the handgrip strength and nutrition indicators of breast cancer patients in relation to HRQoL during treatment.

\section{Materials and Methods}

This was a cross sectional study. This study was conducted at National Cancer Institute, Putrajaya, Malaysia, a national referral center offering various treatments for optimum cancer care in Malaysia (Institut Kanser Negara, 2018), between January and May 2017. The samples were recruited from inpatient oncology wards and daycare center based on purposive sampling. Eligible patients were diagnosed with breast cancer, received at least one month of cancer treatment and were in the midway of receiving treatment. Patients were excluded if they were terminally ill, diagnosed with any psychiatric or neurological disorders. Ethics approval was provided by Medical Research and Ethics Committee (MREC), Ministry of Health Malaysia and Ethics Committee for Research Involving Human Subject Universiti Putra Malaysia. Patients' written informed consents were obtained prior to study enrolment.

\section{Sociodemographic and medical characteristics}

Sociodemographic and medical characteristics of patients were retrieved from the hospital's online information system, otherwise, via interview. Patients' social support and physical activity level were assessed by the modified Medical Outcomes Study Social Support Survey (mMOS-SS) (Moser et al., 2012) and International Physical Activity Questionnaire-short form (IPAQ-SF) (Craig et al., 2003), respectively.

Biochemical data, including serum albumin, hemoglobin, and neutrophils count were obtained from the medical report as secondary data. Weight and height of patients were measured using Dectecto bariatric scale (6857DHR, Webb City, Missouri), with readings taken nearest to $0.1 \mathrm{~kg}$ and $0.1 \mathrm{~cm}$, respectively. Weight status of patient was determined using the universal proxy, body mass index (BMI) according to international cut-off points (World Health Organization. Regional Office for the Western Pacific., 2000). Patients' risk of malnutrition was determined by corrected arm muscle area (CAMA), which was computed from mid-upper arm circumference (MUAC) and triceps skinfold (TSF) thickness (Friedman et al., 1985). MUAC and TSF thickness were measured using Seca Ergonomic circumference measuring tape (201, Detuchland, Germany). With regards to evaluation of physical function, assessment on handgrip strength was ascertained at dominant hands of patients using Lafayette hand dynamometer (78010) and calssified based on age-specific criteria (Chen et al., 2014; Guerra et al., 2014).

\section{Health-related quality of life}

Health-related quality of life (HRQoL) of patients was ascertained using European Organization for Research and Treatment of Cancer quality of life questionnaire Core 30 (EORTC-QLQ-C30) version 3.0. Being a widely used cancer-specific HRQoL questionnaire (Aaronson et al., 1993), EORTC-QLQ-C30 comprises of 30 items, which represents five functional scales (physical, role, emotional, cognitive, and social functioning), three symptom scales (fatigue, nausea, vomiting, and pain), a global health status scale and six single item symptoms (dyspnea, insomnia, appetite loss, constipation, diarrhea, and financial difficulties). Scoring on items of functional and symptoms scales were using four-point Likert scale, ranging from 1 (not at all) to 4 (very much), whereas a seven-point Likert scale was used to rate the items of global health status scale, ranging from 1 (very poor) to 7 (excellent). The mean of component items for each scale was linearly transformed into a range of $0-100$ point, with higher scores indicate better functioning and QoL, but more severe symptoms (Fayers et al., 2001).

\section{Healthy eating index (HEI) - 2015}

A 165 items Malaysian Semi quantitative food frequencies questionnaires (FFQ) was used to assess the habitual dietary intake of patients over the past one month (Institute for Public Health, 2014). Each food item was assigned a standard serving size according to Malaysian Food Album (Malaysian Food Album, 2011). The food consumption data was standardized to frequency consumption of daily basis. The Malaysian food composition database was primarily used for analyzing the nutrient intake via Nutritionist Pro version 4.0. 0 (Axxya system, 2017) with Singaporean and USDA database were the complementary data bases. To assess patients' conformance to dietary guidelines, intakes of patients were transformed into Healthy Eating Index (HEI) scoring (Guenther et al., 2013). The intake of each food component was scored proportionately using the latest version of HEI-2015. The total HEI-2015 score was obtained by summing all individual component score, 
yielding a possible score range of $0-100$, with higher scores indicate a better adherence to Americans' dietary guidelines 2015-2020 (Guenther et al., 2013).

\section{Statistical analysis}

Data analysis was performed with Statistical Software Package (IBM SPSS statistics version 22.0). Mean and standard deviation were presented for normally distributed data. Multiple linear regression was performed to determine the contributions of handgrip strength and nutrition indicators (MUAC, TSF thickness, diet quality, serum albumin, hemoglobin level, and neutrophils count) to primary measure of global health status and other subscales according to potential confounding factors. Variables that were normally distributed for Global Health Status (GHS) and HRQoL subscales (with exception for dyspnea, diarrhea, nausea and vomiting) hence meeting the statistical assumption of using linear regression were selected in the analysis of Multiple Linear Regression. Two multivariate models were performed for each relationship of nutrition indicators and handgrip strength with a series of HRQoL subscales. The first model examined the associations of handgrip strength and each nutrition indicators with HRQoL subscales, adjusting for social support, moderate-to-vigorous physical activity, sociodemographic and medical characteristics. Model 2 examined if there were any changes of associations (resulted in Model 1) after mutually adjusted for nutrition indicators and handgrip strength in the model. All the significant values were set at $p<0.05$.

\section{Results}

\section{Study population}

Of 227 eligible patients approached, 179 patients were consented to participate in the study, giving a total response rate of $79 \%$. In the analysis, 16 patients were excluded due to missing values $(\mathrm{n}=6)$ and extreme outliers $(\mathrm{n}=10)$. A total of 163 patients was included in the final analysis, constituting $69 \%$ of the approached sample.

\section{Subjects' characteristics}

Details for sample are shown in Table 1. Mean age of patients was approximately 50 years (range, 29 - 71 years), with a mean duration of diagnosis at 8.66 months \pm 5.96 . More than half of the patients were diagnosed with advanced cancer (stage III: $n=62 ; 38 \%$; stage IV: $n=41$; $25.2 \%)$ and were currently receiving chemotherapy. The

A

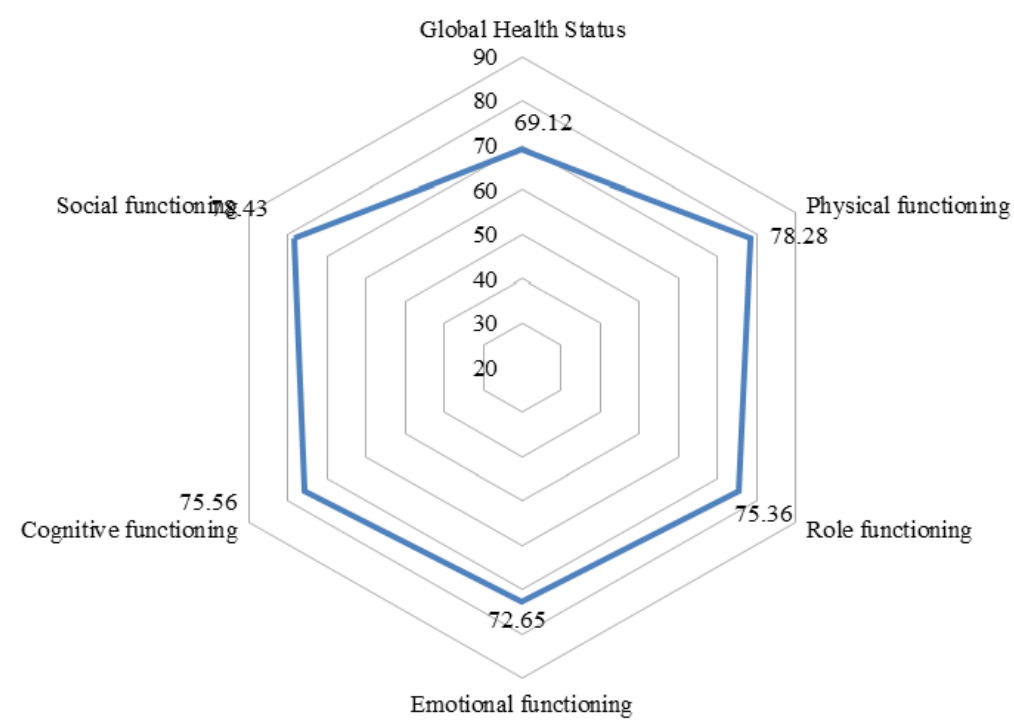

B

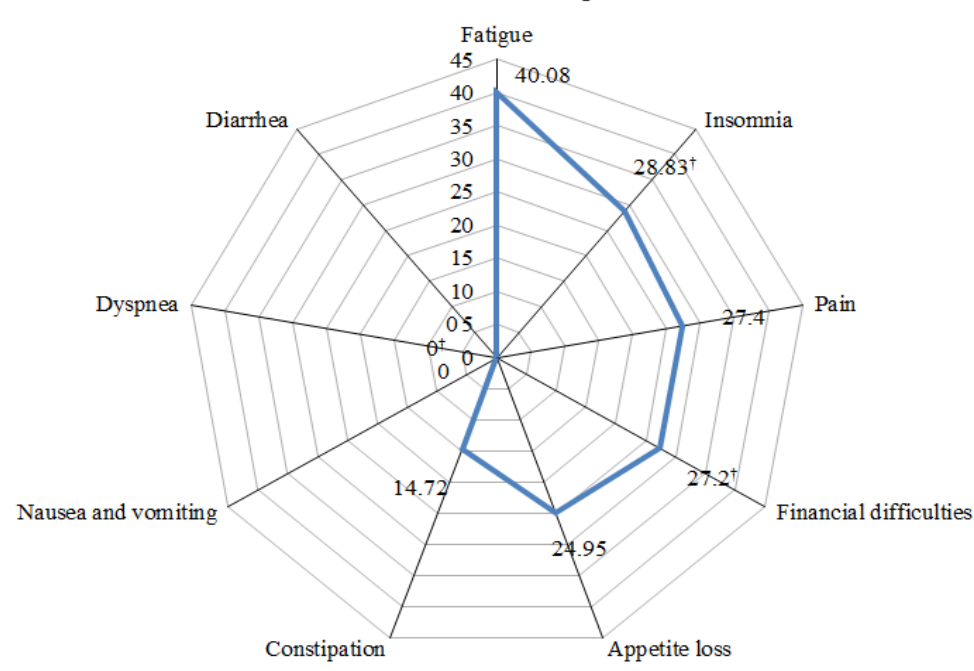

Figure 1. HRQoL Score According to Overall Global Health Status (A), sub-domains of functioning (A) and symptoms (B); †Median 
Table 1. Participants' Characteristics According to Sociodemographic, Medical, Social Support and Physical Activity Level $(\mathrm{N}=163)$

\begin{tabular}{|c|c|c|c|}
\hline & $\mathrm{n}(\%)$ & Mean (SD) & Range \\
\hline Age (years) & & $50.34(10.22)$ & $29.00-71.00$ \\
\hline \multicolumn{4}{|l|}{ Marital status } \\
\hline Single & $16(9.8)$ & & \\
\hline Married & $112(68.7)$ & & \\
\hline Widowed/Separated/Divorced & $35(21.5)$ & & \\
\hline \multicolumn{4}{|l|}{ Educational status } \\
\hline No formal education or primary level & $18(11.0)$ & & \\
\hline Secondary level & $80(49.1)$ & & \\
\hline Tertiary level & $65(39.9)$ & & \\
\hline Being employed & $65(39.9)$ & & \\
\hline Monthly income (RM) & & $1995.66(2163.68)$ & $0-10,000$ \\
\hline Duration of diagnosis (months) & & $8.66(5.96)$ & $1.40-32.15$ \\
\hline \multicolumn{4}{|l|}{ Cancer stage } \\
\hline 0 or I & $6(3.7)$ & & \\
\hline II & $54(33.1)$ & & \\
\hline III & $62(38.0)$ & & \\
\hline IV & $41(25.2)$ & & \\
\hline \multicolumn{4}{|l|}{ Treatment modalities (undergoing) } \\
\hline Chemotherapy & $104(63.8)$ & & \\
\hline Radiotherapy & $34(20.9)$ & & \\
\hline Hormonal therapy & $35(21.5)$ & & \\
\hline Targeted therapy & $25(15.3)$ & & \\
\hline Have any comorbid condition & $72(44.2)$ & & \\
\hline Physical activity level (Total MET-min/week) & & $462(933) \dagger$ & $0-8,200$ \\
\hline Low & $106(65.0)$ & & \\
\hline Moderate & $46(28.2)$ & & \\
\hline High & $11(6.7)$ & & \\
\hline Level of social support & & $82.52(17.26)$ & $21.88-100.00$ \\
\hline Low (1st quartile: <72) & $44(27.0)$ & & \\
\hline Medium $(72-96)$ & $86(52.8)$ & & \\
\hline High (3rd quartile: $\geq 97$ ) & $33(20.2)$ & & \\
\hline Weight (kg) & & $61.78(12.87)$ & $35.60-105.20$ \\
\hline Height (m) & & $155.23(5.48)$ & $140.00-167.00$ \\
\hline BMI $\left(\mathrm{kg} / \mathrm{m}^{2}\right)$ & & $25.63(5.13)$ & $14.92-42.70$ \\
\hline Underweight $(<18.50)$ & $13(8.0)$ & & \\
\hline Normal (18.50 - 24.99) & $66(40.5)$ & & \\
\hline Pre-obese $(25.00-29.99)$ & $50(30.7)$ & & \\
\hline Obese $(\geq 30.00)$ & $34(20.9)$ & & \\
\hline MUAC (cm) & & $29.93(4.97)$ & $18.80-44.95$ \\
\hline TSF thickness (mm) & & $26.46(7.84)$ & $10.70-58.70$ \\
\hline CAMA $\left(\mathrm{cm}^{2}\right)$ & & $31.63(12.26)$ & $6.05-72.78$ \\
\hline$\leq 21.6$ & $33(20.2)$ & & \\
\hline$>21.6$ & $130(79.8)$ & & \\
\hline Serum albumin $(\mathrm{g} / \mathrm{L})$ & & $39.94(3.53)$ & $28.00-50.00$ \\
\hline Normal $(35-50)$ & $152(93.3)$ & & \\
\hline Low $(<35)$ & $11(6.7)$ & & \\
\hline
\end{tabular}


Table 1. Continued

\begin{tabular}{|c|c|c|c|}
\hline & $\mathrm{n}(\%)$ & Mean (SD) & Range \\
\hline Neutrophils count $\left(10^{9} / \mathrm{L}\right)$ & & $3.81(1.85)$ & $0.13-10.24$ \\
\hline Low $(<2.00)$ & $20(12.3)$ & & \\
\hline Normal $(2.00-7.00)$ & $133(81.6)$ & & \\
\hline High $(>7.00)$ & $10(6.1)$ & & \\
\hline Hemoglobin level (g/dL) & & $11.66(1.20)$ & $9.00-15.70$ \\
\hline Normal $(12.00-15.00)$ & $69(42.3)$ & & \\
\hline Low $(<12.00)$ & $93(57.1)$ & & \\
\hline Total HEI-2015 score & & $63.86(8.75)$ & $41.84-88.88$ \\
\hline Good $(>80)$ & $7(4.3)$ & & \\
\hline Needs improvement $(51-80)$ & $146(89.6)$ & & \\
\hline Poor $(<51)$ & $10(6.1)$ & & \\
\hline Handgrip strength (kg) & & $9.60(4.89)$ & $0.00-31.00$ \\
\hline Poor & $163(98.2)$ & & \\
\hline Normal & $3(1.8)$ & & \\
\hline
\end{tabular}

Data were presented as $\mathrm{n}(\%)$ or mean (SD); Ringgit Malaysia exchange rate: 1 MYR, 0.25 USD; Cancer stage: based on anatomic and histological evaluation; †Median (IQR); BMI, body mass index; CAMA, corrected arm muscle area; MUAC, mid-upper arm circumference; TSF thickness, triceps skinfold thickness; HEI, healthy eating index

median MET score was 462 (IQR: 933), with majority of them were physically inactive. With regards to social support level, about half of the patients perceived their social support as average.

Means weight and BMI were $61.78 \mathrm{~kg} \pm 12.87$ and 25.6 $\mathrm{kg} / \mathrm{m}^{2} \pm 5.13$, respectively. Slightly more than half of the patients were overweight, with $\mathrm{BMI} \geq 25 \mathrm{~kg} / \mathrm{m}^{2}$. According to CAMA, an approximately $80 \%$ of patients were well-nourished. Means for serum albumin, neutrophils count, and hemoglobin level were $39.94 \mathrm{~g} / \mathrm{L} \pm 3.53$, $3.81 \times 10^{9} / \mathrm{L} \pm 1.85$ and $11.66 \mathrm{~g} / \mathrm{dL} \pm 1.20$, respectively. Majority of patients had normal serum albumin (93.3\%) and neutrophils count (81.6\%), but with low hemoglobin level $(57.1 \%)$. The mean of total HEI-2015 score was $63.86 \pm 8.75$. More than $90 \%$ of patients had poor diet quality or need dietary modification, with the scores below 80. Patients had mean handgrip strength of $9.60 \mathrm{~kg} \pm 4.89$, with as high as $98.2 \%$ of them had poor muscle strength.

\section{Health-related quality of life}

As a measure for quality of life, the mean score of Global Health Status was 69.12. In term of functional scales, patients scored the highest for social and physical functioning but lowest for emotional functioning (Figure 1A). Among the nine medical symptoms, fatigue (40.08\%) was most commonly experienced by patients, followed by insomnia $(28.83 \%)$, pain $(27.4 \%)$, financial difficulties (27.2\%), and appetite loss (24.95\%). Only a few patients reported nausea and vomiting, dyspnea, and diarrhea (Figure 1B).

\section{Factors associated with Global Health Status}

The results of multiple linear regression models with cancer stage and active chemotherapy as categorical variables were illustrated in Table 2. Breast cancer patients with more advanced stage reported better Global Health Status than those in earlier stage. Patients who were undergoing active chemotherapy were more likely to have worse role function as compared to radiotherapy or other follow-up post-treatment. Tables 3, 4, and 5 demonstrated the multivariate associations of various nutrition indicators and handgrip strength with global health status, functional scales, and medical symptoms. For anthropometric indices, higher MUAC ( $\beta$ : $1.017 ; 95 \%$ CI: $0.35,1.68)$ was related with better GHS after adjusting for social support, physical activity, sociodemographic and medical variables (Model 1). This association remained unchanged after mutually adjusted for nutrition indicators and handgrip strength (Model 2) (Table 2). On the other hand, MUAC was inversely associated with cognitive functioning after adjusting for all the covariates. Higher serum albumin was associated with improved physical functioning and pain (Model 2), but with worse insomnia (Table 3). A positive association of pain was shown with serum hemoglobin, only after controlling for handgrip strength and other nutrition indicators in addition to social support, physical activity, sociodemographic and medical variables (Model 2). Higher serum hemoglobin was associated with improved social functioning, fatigue, and constipation while neutrophils count was the only indicator which significantly associated with constipation (Table 3). Handgrip strength significantly associated with all HRQoL subscales with the exception on constipation and financial difficulties (Table 4).

\section{Discussion}

As nutritional status was a determinant for HRQoL (Lis et al., 2012), present study highlighted the importance of a comprehensive nutritional assessment among breast cancer patients in light of its associations with QoL, functional health, and medical symptoms. Most of the nutrition indicators especially BMI indicated that majority of breast cancer patients were over-nourished. This finding was in agreement with previous studies (Custódio et al., 2016; Fang et al., 2013), which is likely due to inactive 
Table 2. Multivariate Associations of Cancer Stage and Active Chemotherapy with 12 HRQoL Scales

\begin{tabular}{|c|c|c|c|c|c|c|}
\hline \multirow[t]{2}{*}{ Nutritional parameters } & \multicolumn{3}{|c|}{ Cancer stage (III/IV vs 0/I/II) } & \multicolumn{3}{|c|}{ Active chemotherapy (Yes vs No) } \\
\hline & $\beta$ & $P$-value & $95 \% \mathrm{CI}$ & $\beta$ & $P$-value & $95 \% \mathrm{CI}$ \\
\hline \multicolumn{7}{|l|}{ Global Health Status } \\
\hline Model 1 & 7.999 & 0.014 & $(1.65 ; 14.35)$ & -2.019 & 0.572 & $(-9.06 ; 5.02)$ \\
\hline Model 2 & 6.221 & 0.044 & $(0.17,12.28)$ & 0.449 & 0.896 & $(-6.34 ; 7.24)$ \\
\hline \multicolumn{7}{|l|}{ Functional scales } \\
\hline \multicolumn{7}{|l|}{ Physical } \\
\hline Model 1 & -3.647 & 0.245 & $(-9.82 ; 2.52)$ & -1.887 & 0.587 & $(-8.73 ; 4.96)$ \\
\hline Model 2 & -5.022 & 0.064 & $(-10.35 ; 0.30)$ & -1.583 & 0.601 & $(-7.55 ; 4.39)$ \\
\hline \multicolumn{7}{|l|}{ Role } \\
\hline Model 1 & -5.832 & 0.268 & $(-16.20 ; 4.54)$ & -11.795 & 0.045 & $(-23.30 ;-0.29)$ \\
\hline Model 2 & -6.991 & 0.179 & $(-17.22 ; 3.24)$ & -11.681 & 0.046 & $(-23.14 ;-0.22)$ \\
\hline \multicolumn{7}{|l|}{ Emotional } \\
\hline Model 1 & 3.216 & 0.448 & $(-5.15 ; 11.58)$ & 5.245 & 0.266 & $(-4.03 ; 14.52)$ \\
\hline Model 2 & 1.164 & 0.785 & $(-7.24 ; 9.57)$ & 7.505 & 0.117 & $(-1.91 ; 16.93)$ \\
\hline \multicolumn{7}{|l|}{ Cognitive } \\
\hline Model 1 & 4.394 & 0.34 & $(-4.68 ; 13.47)$ & -1.173 & 0.818 & $(-11.24 ; 8.90)$ \\
\hline Model 2 & 2.857 & 0.537 & $(-6.27 ; 11.98)$ & -1.109 & 0.831 & $(-11.34 ; 9.12)$ \\
\hline \multicolumn{7}{|l|}{ Social } \\
\hline Model 1 & -3.295 & 0.491 & $(-12.73 ; 6.14)$ & -8.391 & 0.115 & $(-18.85 ; 2.07)$ \\
\hline Model 2 & -5.168 & 0.274 & $(-14.48 ; 4.14)$ & -4.642 & 0.381 & $(-15.07 ; 5.79)$ \\
\hline \multicolumn{7}{|l|}{ Symptoms scales/items } \\
\hline \multicolumn{7}{|l|}{ Fatigue } \\
\hline Model 1 & -3.116 & 0.441 & $(-11.08 ; 4.85)$ & 7.638 & 0.09 & $(-1.20 ; 16.47)$ \\
\hline Model 2 & -2.789 & 0.485 & $(-10.66 ; 5.08)$ & 5.431 & 0.226 & $(-3.39 ; 14.25)$ \\
\hline \multicolumn{7}{|l|}{ Pain } \\
\hline Model 1 & -1.327 & 0.793 & $(-11.32 ; 8.67)$ & -0.448 & 0.937 & $(-11.54 ; 10.64)$ \\
\hline Model 2 & 0.17 & 0.972 & $(-9.42 ; 9.76)$ & 0.329 & 0.952 & $(-10.42 ; 11.08)$ \\
\hline \multicolumn{7}{|l|}{ Insomnia } \\
\hline Model 1 & -1.827 & 0.77 & $(-14.16 ; 10.51)$ & -7.894 & 0.256 & $(-21.58 ; 5.79)$ \\
\hline Model 2 & 0.718 & 0.908 & $(-11.53 ; 12.97)$ & -6.834 & 0.327 & $(-20.57 ; 6.90)$ \\
\hline \multicolumn{7}{|l|}{ Appetite loss } \\
\hline Model 1 & -5.626 & 0.31 & $(-16.53 ; 5.28)$ & 9.688 & 0.116 & $(-2.41 ; 21.79)$ \\
\hline Model 2 & -5.604 & 0.31 & $(-16.48 ; 5.27)$ & 9.127 & 0.141 & $(-3.06 ; 21.31)$ \\
\hline \multicolumn{7}{|l|}{ Constipation } \\
\hline Model 1 & 0.128 & 0.975 & $(-7.89 ; 8.15)$ & 0.19 & 0.966 & $(-8.71 ; 9.09)$ \\
\hline Model 2 & 1.452 & 0.719 & $(-6.50 ; 9.40)$ & -3.497 & 0.439 & $(-12.41 ; 5.41)$ \\
\hline \multicolumn{7}{|l|}{ Financial difficulties } \\
\hline Model 1 & -0.085 & 0.988 & $(-11.54 ; 11.37)$ & -4.552 & 0.48 & $(-17.26 ; 8.16)$ \\
\hline Model 2 & 0.706 & 0.907 & $(-11.19 ; 12.60)$ & -4.967 & 0.463 & $(-18.30 ; 8.36)$ \\
\hline
\end{tabular}

Model 1, Adjusted for age, being employed, monthly income, married, educational level (secondary, tertiary or primary, no formal education) + cancer stage (III, IV or 0, I, II), time since diagnosis, chemotherapy, comorbidity + social support + moderate/vigorous physical activity level; Model 2, Model $1+$ nutrition indicators + handgrip strength Figures in bold indicate statistically significant values $(\mathrm{p}<0.05$ )

lifestyle resulted in energy imbalance (Vance et al., 2011), partially attributed to the high prevalence of physically inactive among the patients. More studies are warranted as present study did not determine the changes of physical activity level before and during treatment. With respect to cancer severity, a study revealed a total of $51.4 \%$, $48.8 \%$, and $46.1 \%$ of breast cancer patients with stage I, II, and III, respectively practiced moderate-to-vigorous physical activity level (20.2 MET-hours) during treatment, however, the strength of association between cancer stage and physical activity was weak (Mandelblatt et al., 2011). It is reasonable that overweight in breast cancer stem from climate factor (Von Hippel and Benson, 2014), as this study was conducted during hot session which may discourage the patients to carry out physical activity, especially outdoor activities. On the other hand, hemoglobin level 
Table 3. Multivariate Associations of Anthropometric and Dietary Indicators with 12 HRQoL Scales

\begin{tabular}{|c|c|c|c|c|c|c|c|c|c|}
\hline \multirow[t]{2}{*}{ Nutritional parameters } & \multicolumn{3}{|c|}{ MUAC } & \multicolumn{3}{|c|}{ TSF thickness } & \multicolumn{3}{|c|}{ HEI-2015 } \\
\hline & $\beta$ & $P$-value & $95 \% \mathrm{CI}$ & $\beta$ & $P$-value & $95 \% \mathrm{CI}$ & $\beta$ & $P$-value & $95 \% \mathrm{CI}$ \\
\hline \multicolumn{10}{|l|}{ Global Health Status } \\
\hline Model 1 & 1.017 & 0.003 & $(0.35,1.68)$ & 0.241 & 0.254 & $(-0.18,0.66)$ & -0.124 & 0.51 & $(-0.49,0.25)$ \\
\hline Model 2 & 1.277 & 0.014 & $(0.27,2.29)$ & -0.356 & 0.247 & $(-0.96,0.25)$ & -0.095 & 0.597 & $(-0.45,0.26)$ \\
\hline \multicolumn{10}{|l|}{ Functional scales } \\
\hline \multicolumn{10}{|l|}{ Physical } \\
\hline Model 1 & 0.156 & 0.642 & $(-0.51,0.82)$ & 0.127 & 0.535 & $(-0.28,0.53)$ & 0.264 & 0.145 & $(-0.09,0.62)$ \\
\hline Model 2 & -0.332 & 0.465 & $(-1.23,0.56)$ & 0.347 & 0.203 & $(-0.19,0.88)$ & 0.202 & 0.206 & $(-0.11,0.52)$ \\
\hline \multicolumn{10}{|l|}{ Role } \\
\hline Model 1 & -0.372 & 0.5 & $(-1.46,0.72)$ & -0.17 & 0.613 & $(-0.84,0.49)$ & 0.209 & 0.484 & $(-0.38,0.80)$ \\
\hline Model 2 & -0.957 & 0.261 & $(-2.64,0.72)$ & 0.338 & 0.508 & $(-0.67,1.34)$ & 0.07 & 0.814 & $(-0.52,0.66)$ \\
\hline \multicolumn{10}{|l|}{ Emotional } \\
\hline Model 1 & -0.395 & 0.387 & $(-1.30,0.51)$ & -0.493 & 0.076 & $(-1.04,0.05)$ & 0.016 & 0.947 & $(-0.47,0.50)$ \\
\hline Model 2 & 0.222 & 0.756 & $(-1.19,1.63)$ & -0.629 & 0.144 & $(-1.48,0.22)$ & 0.058 & 0.816 & $(-0.44,0.55)$ \\
\hline \multicolumn{10}{|l|}{ Cognitive } \\
\hline Model 1 & -0.715 & 0.146 & $(-1.68,0.25)$ & -0.177 & 0.557 & $(-0.77,0.42)$ & -0.194 & 0.466 & $(-0.72,0.33)$ \\
\hline Model 2 & -1.543 & 0.048 & $\begin{array}{l}(-3.07, \\
-0.01)\end{array}$ & 0.553 & 0.235 & $(-0.36,1.47)$ & -0.283 & 0.299 & $(-0.82,0.25)$ \\
\hline \multicolumn{10}{|l|}{ Social } \\
\hline Model 1 & 0.112 & 0.827 & $(-0.90,1.23)$ & 0 & 0.999 & $(-0.62,0.62)$ & -0.348 & 0.21 & $(-0.89,0.20)$ \\
\hline Model 2 & -0.231 & 0.77 & $(-1.79,1.33)$ & 0.083 & 0.862 & $(-0.85,1.02)$ & -0.497 & 0.075 & $(-1.05,0.05)$ \\
\hline \multicolumn{10}{|l|}{ Symptoms scales/items } \\
\hline \multicolumn{10}{|l|}{ Fatigue } \\
\hline Model 1 & 0.469 & 0.278 & $(-0.38,1.32)$ & 0.42 & 0.11 & $(-0.10,0.94)$ & -0.41 & 0.077 & $(-0.87,0.05)$ \\
\hline Model 2 & 0.017 & 0.979 & $(-1.29,1.33)$ & 0.402 & 0.313 & $(-0.38,1.19)$ & -0.298 & 0.202 & $(-0.76,0.16)$ \\
\hline \multicolumn{10}{|l|}{ Pain } \\
\hline Model 1 & -0.389 & 0.472 & $(-1.46,0.68)$ & -0.32 & 0.332 & $(-0.97,0.33)$ & -0.45 & 0.122 & $(-1.02,0.12)$ \\
\hline Model 2 & 0.221 & 0.786 & $(-1.38,1.82)$ & -0.476 & 0.329 & $(-1.44,0.49)$ & -0.491 & 0.087 & $(-1.06,0.07)$ \\
\hline \multicolumn{10}{|l|}{ Insomnia } \\
\hline Model 1 & -0.814 & 0.224 & $(-2.13,0.50)$ & -0.28 & 0.493 & $(-1.09,0.53)$ & 0.012 & 0.974 & $(-0.70,0.73)$ \\
\hline Model 2 & -0.235 & 0.82 & $(-2.27,1.80)$ & -0.152 & 0.807 & $(-1.37,1.07)$ & -0.199 & 0.584 & $(-0.92,0.52)$ \\
\hline \multicolumn{10}{|l|}{ Appetite loss } \\
\hline Model 1 & -0.625 & 0.287 & $(-1.78,0.53)$ & -0.34 & 0.343 & $(-1.05,0.37)$ & -0.523 & 0.099 & $(-1.14,0.10)$ \\
\hline Model 2 & -0.413 & 0.652 & $(-2.22,1.39)$ & -0.206 & 0.707 & $(-1.29,0.88)$ & -0.444 & 0.169 & $(-1.08,0.19)$ \\
\hline \multicolumn{10}{|l|}{ Constipation } \\
\hline Model 1 & 0.252 & 0.565 & $(-0.61,1.12)$ & 0.098 & 0.713 & $(-0.43,0.63)$ & 0.153 & 0.517 & $(-0.31,0.62)$ \\
\hline Model 2 & 0.128 & 0.849 & $(-1.20,1.46)$ & 0.11 & 0.786 & $(-0.69,0.91)$ & 0.206 & 0.385 & $(-0.26,0.67)$ \\
\hline \multicolumn{10}{|l|}{ Financial difficulties } \\
\hline Model 1 & 0.297 & 0.635 & $(-0.94,1.53)$ & -0.022 & 0.954 & $(-0.78,0.73)$ & 0.207 & 0.541 & $(-0.46,0.87)$ \\
\hline Model 2 & 0.852 & 0.401 & $(-1.15,2.85)$ & -0.372 & 0.541 & $(-1.57,0.83)$ & 0.212 & 0.552 & $(-0.49,0.91)$ \\
\hline
\end{tabular}

Model 1, Adjusted for age, being employed, monthly income, married, educational level (secondary, tertiary, or primary or no formal education) + cancer stage (III, IV, or 0, I or II), time since diagnosis, chemotherapy, comorbidity + social support + moderate/vigorous physical activity level; Model 2, Model $1+$ nutrition indicators + handgrip strength Figures in bold indicate statistically significant values ( $\mathrm{p}<0.05$ )

was slightly lower than the recommendation. In addition to nutritional issues, the abnormally low hemoglobin level could be manifested by disease- or treatment-related factors (Hidding et al., 2014; Naoum, 2016), elucidating a low specificity in respect of the nutritional prognosis. Therefore, a multimodal of nutritional assessment should be taken into consideration to ensure diagnostic accuracy.
With regards to HRQoL pattern, present study indicated an average score of overall QoL, which is comparable with previous studies (Høyer et al., 2011; Ng et al., 2015; Rohani et al., 2015). On the other hand, varied distressing symptoms and functional limitations were widely reported across the studies (Høyer et al., 2011; Ng et al., 2015; Rohani et al., 2015). Discrepancies of HRQoL 
Table 4. Multivariate Associations of Biochemical Indicators with 12 HRQoL Scales

\begin{tabular}{|c|c|c|c|c|c|c|c|c|c|}
\hline \multirow{2}{*}{$\begin{array}{l}\text { Nutritional } \\
\text { parameters }\end{array}$} & \multicolumn{3}{|c|}{ Serum albumin } & \multicolumn{3}{|c|}{ Serum hemoglobin } & \multicolumn{3}{|c|}{ Neutrophils count } \\
\hline & $\beta$ & $P$-value & $95 \% \mathrm{CI}$ & $\beta$ & $P$-value & $95 \% \mathrm{CI}$ & $\beta$ & $P$-value & $95 \% \mathrm{CI}$ \\
\hline \multicolumn{10}{|c|}{ Global Health Status } \\
\hline Model 1 & -0.182 & 0.761 & $(-1.05,0.77)$ & 1.217 & 0.357 & $(-1.39,3.82)$ & 0.527 & 0.539 & $(-1.16,2.22)$ \\
\hline Model 2 & -0.133 & 0.765 & $(-1.02,0.75)$ & 1.391 & 0.292 & $(-1.21,4.00)$ & 0.671 & 0.418 & $(-0.96,2.31)$ \\
\hline \multicolumn{10}{|c|}{ Functional scales } \\
\hline \multicolumn{10}{|l|}{ Physical } \\
\hline Model 1 & 1.626 & $<0.001$ & $(0.80,2.45)$ & 0.037 & 0.977 & $(-2.50,2.57)$ & -0.198 & 0.811 & $(-1.84,1.44)$ \\
\hline Model 2 & 1.694 & $<0.001$ & $(0.91,2.48)$ & -2.174 & 0.064 & $(-4.47,0.13)$ & 0.484 & 0.509 & $(-0.96,1.93)$ \\
\hline \multicolumn{10}{|l|}{ Role } \\
\hline Model 1 & 1.143 & 0.112 & $(-0.27,2.56)$ & 0.515 & 0.807 & $(-3.64,4.67)$ & -1.358 & 0.319 & $(-4.04,1.33)$ \\
\hline Model 2 & 0.927 & 0.213 & $(-0.54,2.39)$ & -0.754 & 0.73 & $(-5.07,3.56)$ & -1.012 & 0.462 & $(-3.73,1.70)$ \\
\hline \multicolumn{10}{|l|}{ Emotional } \\
\hline Model 1 & -0.303 & 0.613 & $(-1.48,0.88)$ & 0.287 & 0.869 & $(-3.16,3.73)$ & 1.451 & 0.198 & $(-0.77,3.67)$ \\
\hline Model 2 & -0.328 & 0.599 & $(-1.56,0.90)$ & 0.109 & 0.953 & $(-3.52,3.73)$ & 1.793 & 0.122 & $(-0.49,4.07)$ \\
\hline \multicolumn{10}{|l|}{ Cognitive } \\
\hline Model 1 & 0.761 & 0.237 & $(-0.51,2.03)$ & -0.44 & 0.815 & $(-4.15,3.27)$ & 0.241 & 0.843 & $(-2.16,2.65)$ \\
\hline Model 2 & 0.713 & 0.292 & $(-0.62,2.05)$ & -1.381 & 0.488 & $(-5.31,2.55)$ & 0.183 & 0.884 & $(-2.29,2.06)$ \\
\hline \multicolumn{10}{|l|}{ Social } \\
\hline Model 1 & 0.321 & 0.633 & $(-1.01,1.65)$ & 4.269 & 0.028 & $(0.46,8.08)$ & 0.348 & 0.784 & $(-2.16,2.85)$ \\
\hline Model 2 & -0.099 & 0.885 & $(-1.46,1.26)$ & 4.615 & 0.024 & $(0.61,8.63)$ & -0.214 & 0.867 & $(-2.74,2.31)$ \\
\hline \multicolumn{10}{|c|}{ Symptoms scales/items } \\
\hline \multicolumn{10}{|l|}{ Fatigue } \\
\hline Model 1 & -0.644 & 0.254 & $(-1.76,0.47)$ & -3.554 & 0.03 & $(-6.76,-0.35)$ & 0.351 & 0.743 & $(-1.76,2.46)$ \\
\hline Model 2 & -0.174 & 0.764 & $(-1.32,0.97)$ & -2.963 & 0.084 & $(-6.37,0.40)$ & 0.103 & 0.923 & $(-2.01,2.22)$ \\
\hline \multicolumn{10}{|l|}{ Pain } \\
\hline Model 1 & -1.377 & 0.05 & $(-2.76,0.00)$ & 1.452 & 0.482 & $(-2.62,5.52)$ & -0.993 & 0.457 & $(-3.63,1.64)$ \\
\hline Model 2 & -1.672 & 0.019 & $(-3.07,-0.27)$ & 4.285 & 0.042 & $(0.17,8.40)$ & -1.987 & 0.132 & $(-4.58,0.60)$ \\
\hline \multicolumn{10}{|l|}{ Insomnia } \\
\hline Model 1 & 1.837 & 0.035 & $(0.14,3.54)$ & 4.969 & 0.05 & $(-0.01,9.95)$ & -1.43 & 0.387 & $(-4.69,1.83)$ \\
\hline Model 2 & 1.37 & 0.13 & $(-0.41,3.15)$ & 4.79 & 0.073 & $(-0.44,10.03)$ & -1.921 & 0.251 & $(-5.22,1.37)$ \\
\hline \multicolumn{10}{|l|}{ Appetite loss } \\
\hline Model 1 & -1.444 & 0.059 & $(-2.94,0.06)$ & -2.472 & 0.27 & $(-6.88,1.94)$ & 0.605 & 0.677 & $(-2.26,3.47)$ \\
\hline Model 2 & -1.285 & 0.109 & $(-2.86,0.29)$ & -0.7 & 0.766 & $(-5.34,3.94)$ & 0.064 & 0.965 & $(-2.85,2.98)$ \\
\hline \multicolumn{10}{|l|}{ Constipation } \\
\hline Model 1 & -0.529 & 0.355 & $(-1.66,0.60)$ & -4.609 & 0.005 & $(-7.82,-1.40)$ & -2.761 & 0.01 & $(-4.85,-0.68)$ \\
\hline Model 2 & -0.246 & 0.676 & $(-1.41,0.92)$ & -4.053 & 0.021 & $(-7.48,-0.63)$ & -2.43 & 0.027 & $(-4.58,-0.28)$ \\
\hline \multicolumn{10}{|c|}{ Financial difficulties } \\
\hline Model 1 & 0.287 & 0.726 & $(-1.33,1.90)$ & -0.543 & 0.82 & $(-5.26,4.17)$ & -1.526 & 0.323 & $(-4.57,1.52)$ \\
\hline Model 2 & 0.352 & 0.691 & $(-1.39,2.09)$ & -0.53 & 0.838 & $(-5.66,4.60)$ & -1.164 & 0.477 & $(-4.39,2.07)$ \\
\hline
\end{tabular}

Model 1, Adjusted for age, being employed, monthly income, married, educational level (secondary, tertiary, or primary or no formal education) + cancer stage (III, IV, or 0, I or II), time since diagnosis, chemotherapy, comorbidity + social support + moderate/vigorous physical activity level; Model 2, Model $1+$ nutrition indicators + handgrip strength Figures in bold indicate statistically significant values $(\mathrm{p}<0.05$ )

between countries could be explained by the variation in social cultural perspective as well as the economy status. The scores for certain symptoms among the patients in this study were zero when compared to the EORTC-QLQ-C30 population reference values (Scott et al., 2008). Likewise, nausea and vomiting, dyspnea, and diarrhea were the least complaint symptoms in present study, which explained the asymmetric radar chart. It should be noted that HRQoL is highly dependent on personal values or priorities, whereby each domain could be perceived differently across individual as well as population.

Unexpectedly, patients with higher grade of breast cancer perceived better overall QoL during treatment. The result of Iran study revealed that breast cancer patients with more advanced cancer were likely to experience worse QoL, in the aspects of physical and role 
Table 5. Multivariate Associations of Handgrip Strength with 12 HRQoL Scales

\begin{tabular}{|c|c|c|c|}
\hline & \multicolumn{3}{|c|}{ Handgrip strength } \\
\hline & $\beta$ & $P$-value & $95 \% \mathrm{CI}$ \\
\hline \multicolumn{4}{|c|}{ Global Health Status } \\
\hline Model 1 & 1.382 & $<0.001$ & $(0.77,2.00)$ \\
\hline Model 2 & 1.215 & $<0.001$ & $(0.59,1.84)$ \\
\hline \multicolumn{4}{|c|}{ Functional scales } \\
\hline \multicolumn{4}{|l|}{ Physical } \\
\hline Model 1 & 1.751 & $<0.001$ & $(1.18,2.32)$ \\
\hline Model 2 & 1.782 & $<0.001$ & $(1.23,2.34)$ \\
\hline \multicolumn{4}{|l|}{ Role } \\
\hline Model 1 & 1.788 & 0.001 & $(0.79,2.79)$ \\
\hline Model 2 & 1.88 & $<0.001$ & $(0.84,2.92)$ \\
\hline \multicolumn{4}{|l|}{ Emotional } \\
\hline Model 1 & 1.038 & 0.017 & $(0.19,1.89)$ \\
\hline Model 2 & 1.031 & 0.021 & $(0.16,1.91)$ \\
\hline \multicolumn{4}{|l|}{ Cognitive } \\
\hline Model 1 & 0.877 & 0.062 & $(-0.04,1.80)$ \\
\hline Model 2 & 1.11 & 0.022 & $(0.16,2.06)$ \\
\hline \multicolumn{4}{|l|}{ Social } \\
\hline Model 1 & 1.441 & 0.003 & $(0.50,2.38)$ \\
\hline Model 2 & 1.447 & 0.004 & $(0.48,2.41)$ \\
\hline \multicolumn{4}{|c|}{ Symptoms scales/items } \\
\hline \multicolumn{4}{|l|}{ Fatigue } \\
\hline Model 1 & -1.164 & 0.004 & $(-1.96,-0.37)$ \\
\hline Model 2 & -1.092 & 0.009 & $(-1.90,-0.28)$ \\
\hline \multicolumn{4}{|l|}{ Pain } \\
\hline Model 1 & -1.703 & 0.001 & $(-2.69,-0.72)$ \\
\hline Model 2 & -1.761 & 0.001 & $(-2.75,-0.77)$ \\
\hline \multicolumn{4}{|l|}{ Insomnia } \\
\hline Model 1 & -1.61 & 0.011 & $(-2.85,-0.37)$ \\
\hline Model 2 & -1.705 & 0.008 & $(-2.97,-0.44)$ \\
\hline \multicolumn{4}{|l|}{ Appetite loss } \\
\hline Model 1 & -1.423 & 0.011 & $(-2.51,-0.34)$ \\
\hline Model 2 & -1.303 & 0.023 & $(-2.42,-0.19)$ \\
\hline \multicolumn{4}{|l|}{ Constipation } \\
\hline Model 1 & -0.49 & 0.241 & $(-1.31,0.33)$ \\
\hline Model 2 & -0.509 & 0.225 & $(-1.33,0.32)$ \\
\hline \multicolumn{4}{|c|}{ Financial difficulties } \\
\hline Model 1 & -0.13 & 0.828 & $(-1.31,1.05)$ \\
\hline Model 2 & -0.289 & 0.645 & $(-1.53,0.95)$ \\
\hline
\end{tabular}

Model 1, Adjusted for age, being employed, monthly income, married, educational level (secondary, tertiary, or primary or no formal education) + cancer stage (III, IV, or 0, I or II), time since diagnosis, chemotherapy, comorbidity + social support + moderate/vigorous physical activity level; Model 2, Model $1+$ nutrition indicators Figures in bold indicate statistically significant values $(\mathrm{p}<0.05)$

functioning (Rohani et al., 2015). With the continuity of active treatment, a majority of patients especially those diagnosed with advanced cancer received palliative care for symptom relief purpose (Kelley and Meier, 2010). This may elucidate the benefit of initiating palliative care for cancer HRQoL while receiving aggressive cancer treatments. In comparison with other treatments including of radiotherapy, hormonal therapy, and targeted therapy, chemotherapy resulted in poorer role functioning among breast cancer patients. Chemotherapy drugs attack the cancer cells by entering the blood circulation, which could also damage the healthy cells, resulting in symptoms of altered appetite, nausea and vomiting (American Cancer Society, 2016), which could affect patients' ability to perform daily activities and their HRQoL.

With the exceptions of constipation and financial difficulties, higher handgrip strength was consistently associated with better HRQoL. Highly related with impaired physical function and overall QoL (Christensen et al., 2014; Kilgour et al., 2013), impaired muscle function is evident among breast cancer patients (Hidding et al., 2014), which is attributable to lymph node dissection or cancer treatment (Hidding et al., 2014). The assessment of handgrip strength reflects well the strength of arm muscles, in relation to physical function. Despite the utilization of handgrip strength remains low in clinical setting, it can be considered as a good proxy for physical function evaluation, which is recognized as one of the major functional components for HRQoL evaluation. Handgrip strength shows incompatible result with body composition indices in regard to the evaluation of nutritional status, elucidating that it is a relatively weak indicator of muscle wasting or malnutrition (Shi and Chen, 2017) for breast cancer patients during treatment. Benavides-Rodríguez et al., (2017) had previously demonstrated an inverse association between handgrip strength and body mass index in breast cancer survivors (Benavides-Rodríguez et al., 2017). More studies are needed to delineate the significant role of handgrip strength measurement among this population, particularly on-treatment phase.

On the other hand, all nutrition indicators except TSF thickness and diet quality were found to be associated significantly with various aspects of HRQoL. In corroborating with previous studies (Rahman et al., 2014; Xia et al., 2018), current finding revealed that overall QoL was improved with MUAC, which is a good surrogate of BMI (Brito et al., 2016). As MUAC and BMI have strong multicollinearity, MUAC that explained better the HRQoL outcomes was selected in the final regression model. Earlier systematic review supported the relationship of HRQoL with nutritional status (Lis et al., 2012). Treatment-induced side effects predispose cancer patients to poor oral intake and under-nutrition, which negatively affect their HRQoL, particularly for physical and emotional function (Arends et al., 2017). However, the direction of relationship between HRQoL and nutritional status remains unanswered. Studies on breast cancer demonstrated negative impact of being obesity on HRQoL (Doll et al., 2015; Fang et al., 2013; Paxton et al., 2012), where poorer HRQoL was found with higher body fat level (Frenzel et al., 2013). As seen in the present study, reduced cognitive function was shown as MUAC increases, which could be explained by the physiology of obesity induced cognitive impairment (O'Brien et al., 2017). As there is little evidence about the relationship of body composition with HRQoL, more studies are 
deemed necessary to prove this notion. It is noteworthy that muscle mass and body fat percentage of breast cancer patients were assessed. Inconsistent with previous studies (George et al., 2014; Wayne et al., 2006), diet quality did not show any significant finding with HRQoL. This could be due to different instruments used to assess diet quality and HRQoL.

All biochemical indicators were shown to contribute to better HRQoL, except for insomnia and pain scales. Although higher serum albumin was significantly associated with worse insomnia, this relationship became attenuated when controlling for nutrition indicators and handgrip strength (Model 2). This suggests that nutrition indicators or handgrip strength could be potential confounders of the relationship between serum albumin and insomnia. Romero-Corral et al., (2010) proposed that being obesity is the major contributor of obstructive sleep apnea, as manifested by symptom of insomnia (Romero-Corral et al., 2010). Blood transfusion is commonly used to manage cancer-related anemia, which may explain the finding of positive relationship between serum hemoglobin and pain. A result of breast cancer study revealed that higher serum hemoglobin resulted in improved fatigue and functional capacity (Jacobsen et al., 2004), which was in line with present study. Hemoglobin is a type of protein that carry and supply oxygen for cellular activities, which related closely with fatigue. In view of the interrelation among symptoms, functional health, and overall QoL (Ferrans et al., 2005), present finding suggests the possible negative impact of low serum hemoglobin on social functioning by inducing fatigue. As shown in the finding, patients with low neutrophils count were likely to experience constipation. An abnormally low neutrophils count should be paid with great attention as it might indicate higher risk of getting constipation induced-infection, which could be life-threatening (Kawsar et al., 2012). There was a substantial proportion of patients diagnosed with high graded cancer and receiving chemotherapy, reflecting the studied population are weak or vulnerable. As disease and treatment-related factors pose substantially effect on patients' HRQoL, the main essence of study in determining the association between nutrition indicators and physical function with HRQoL could be affected. Despite these two factors were controlled for multivariate analysis, the finding should be interpreted cautiously.

The strengths of the present study include the exploration of nutrition indicators, which were in relation to multi-aspects of HRQoL, accounting for various potential confounders. However, as this study was a cross sectional design in nature, hence the causality of association between nutritional factors and HRQoL could not be confirmed. Lack of control group as a point of comparison may diminish the ability to identify the extent to which breast cancer patients perceived their HRQoL during treatment, whereby a case control study should be initiated in the future. On the other hand, as this study recruited patients from a single institution, the generalization of study population could not be precluded. There is possibility of eliminating patients with emotional distress or impaired HRQoL, given that the participation of this study was on voluntary basis. Moreover, the current finding may underestimate the symptoms induced by cancer treatment due to large gap between treatment and assessment days.

The physical measure of handgrip strength was the most prominent indicator for most of the HRQoL outcomes. Handgrip strength is considered as the recognized measure used for HRQoL evaluation, particularly in the aspect of physical function. A good nutritional status was associated with improved overall QoL. Current finding depicts potential benefits of being well-nourished and having stronger muscle on HRQoL outcomes among breast cancer patients undergoing treatment, which highlights the need for appropriate nutritional intervention to improve HRQoL among breast cancer patients.

\section{Acknowledgements}

Special thanks are dedicated to all patients and staffs of National Cancer Institute at Putrajaya, for the enthusiasm in data collection. The authors thank Dr Subashini Elangkovan from the Clinical Research Centre; Norshariza Jamhuri, Betti Sharina Mohd Haniff, Siti Nuraini Mohd Samwil, and $\mathrm{Ng}$ Wai Han from the Department of Dietetics, for assistance along the data collection.

\section{Funding Statement}

This work was supported by the University Putra Malaysia (grant project number GP-IPS/2016/ 9507400).

\section{References}

Aaronson NK, Ahmedzai S, Bergman B, et al (1993). The European organization for research and treatment of cancer QLQ-C30: A quality-of-life instrument for use in international clinical trials in oncology. J Nat Cancer Instit, 85, 365-6.

American Cancer Society (2016). Breast Cancer detailed guide. Cancer Org, pp 1-127. https://doi.org/10.1634/ Theoncologist.2016-0067

Arends J, Baracos V, Bertz H, et al (2017). ESPEN expert group recommendations for action against cancer-related malnutrition. Clin Nutr, 36, 1187-96.

Benavides-Rodríguez L, García-Hermoso A, Rodrigues-Bezerra D, et al (2017). Relationship between handgrip strength and muscle mass in female survivors of breast cancer: A mediation analysis. Nutrients, 9, 1-14.

Boltong A, Aranda S, Keast R, et al (2014). A prospective cohort study of the effects of adjuvant breast cancer chemotherapy on taste function, food liking, appetite and associated nutritional outcomes. PLoS One, 9, 1-9.

Brito NB, Suárez Llanos JP, Ferrer MF, et al (2016). Relationship between mid-upper arm circumference and body mass index in inpatients. PLoS One, 11, 1-10.

Chen LK, Liu LK, Woo J, et al (2014). Sarcopenia in Asia: Consensus report of the Asian working group for sarcopenia. $J$ Am Med Directors Assoc, Elsevier Ltd.

Christensen JF, Jones LW, Andersen JL, et al (2014). Muscle dysfunction in cancer patients. Ann Oncol, Official Journal of the European Society for Medical Oncology / ESMO.

Craig CL, Marshall AL, Sjöström M, et al (2003). International physical activity questionnaire: 12-Country reliability and validity. Med Sci Sports Exerc, 35, 1381-95.

Custódio IDD, Marinho EDC, Gontijo CA, et al (2016). Impact 
of chemotherapy on diet and nutritional status of women with breast cancer: A prospective study. PLoS One, 11, 1-21.

Dang CC, Wong KZ, Lim M, Zulkefle N (2016). Health related quality of life (HRQoL) among breast cancer patients receiving chemotherapy in Hospital Melaka: Single Centre Experience. Asian Pac J Cancer Prev, 17, 5121-6.

de Vries YC, van den Berg MMGA, de Vries JHM, et al (2017). Differences in dietary intake during chemotherapy in breast cancer patients compared to women without cancer. Supportive Care Cancer, 25, 2581-91.

Doll KM, Kalinowski AK, Snavely AC, et al (2015). Obesity is associated with worse quality of life in women with gynecologic malignancies: An opportunity to improve patient-centered outcomes. Cancer, 121, 395-402.

Fang P, Tan KS, Troxel AB, et al (2013). High body mass index is associated with worse quality of life in breast cancer patients receiving radiotherapy. Breast Cancer Res Treat, 141, 125-33.

Fayers P, Aaronson NK, Bjordal K, et al (2001). EORTC QLQ-C30 Scoring Manual (3rd ed., Vol. 30). Eur Organ Res Treat Cancer, Brussels. Retrieved from https://www.eortc. org/app/uploads/sites/2/2018/02/SCmanual.pdf.

Ferlay J, Soerjomataram I, Ervik M, et al (2014). GLOBOCAN 2012 v1. 0, Cancer incidence and mortality worldwide: IARC CancerBase No. 11. 2013.

Ferrans CE, Zerwic JJ, Wilbur JE, Larson JL (2005). Conceptual model of health-related quality of life. Journal of Nursing Scholarship: An Official Publication of Sigma Theta Tau International Honor Society of Nursing / Sigma Theta Tau, 37, 336-42.

Fiteni F, Westeel V, Pivot X, et al (2014). Endpoints in cancer clinical trials. J Visc Surg, 151, 17-22.

Frenzel A, Pastore C, González MC (2013). The influence of body composition on quality of life of patients with breast cancer. Nutrición Hospitalaria, 28, 1475-82.

Friedman PJ, Campbell AJ, Caradoc-davies TH (1985). Prospective trial of a new diagnostic criterion for severe wasting malnutrition in the elderly. Age Ageing, 14, 149-54.

George SM, Alfano CM, Neuhouser ML, et al (2014). Better postdiagnosis diet quality is associated with less cancerrelated fatigue in breast cancer survivors. J Cancer Surv, 8, 680-7.

Guenther PM, Casavale KO, Kirkpatrick SI, et al (2013). Update of Healthy Eating Index: HEI-2010. J Acad Nur Diet, 113, $1-20$.

Guerra RS, Fonseca I, Pichel F, Restivo MT, Amaral TF (2014). Handgrip strength cutoff values for undernutrition screening at hospital admission. Eur J Clin Nutr, 68, 1315-21.

Hidding, Beurskens, Wees, et al (2014). Treatment Related Impairments in Arm and Shoulder in Patients with Breast Cancer: A Systematic Review. PLoS One, 9, e96748.

Høyer M, Johansson B, Nordin K, et al (2011). Health-related quality of life among women with breast cancer - a population-based study. Acta Oncol, 50, 1015-26.

Institut Kanser Negara (2018). Profile. Retrieved February 18, 2018, from http://nci.moh.gov.my/index.php/ms/info/profile

Institute for Public Health (2014). National Health and Morbidity Survey 2014: Malaysian Adult Nutrition Survey Volume I: Methodology and General Findings.

Jacobsen PB, Garland LL, Booth-Jones M, et al (2004). Relationship of hemoglobin levels to fatigue and cognitive functioning among cancer patients receiving chemotherapy. J Pain Symptom Manage, 28, 7-18.

Kane HL, Halpern MT, Squiers LB, Treiman KA, McCormack LA (2014). Implementing and evaluating shared decision making in oncology practice. CA Cancer J Clin, 64, 377-88.

Kawsar HI, Gopal KV, Shahnewaz J, Daw HA (2012).
Constipation in clostridium difficile infection. BMJ Case Rep, bcr0220125938, 2-4.

Kelley AS, Meier D (2010). Palliative care - A Shifting Paradigm. N Engl J Med, 363, 781-2.

Kilgour RD, Vigano A, Trutschnigg B, et al (2013). Handgrip strength predicts survival and is associated with markers of clinical and functional outcomes in advanced cancer patients. Supportive Care Cancer, 21, 3261-70.

Lis CG, Gupta D, Lammersfeld CA, Markman M, Vashi PG (2012). Role of nutritional status in predicting quality of life outcomes in cancer--a systematic review of the epidemiological literature. Nutr $J, \mathbf{1 1}, 27$.

Malaysian Food Album (2011). Institute for Public Health (IPH).

Mandelblatt JS, Luta G, Kwan ML, et al (2011). Associations of physical activity with quality of life and functional ability in breast cancer patients during active adjuvant treatment: The Pathways Study. Breast Cancer Res Treat, 129, 521-9.

Moser A, Stuck AE, Silliman RA, Ganz PA, Clough-Gorr KM (2012). The eight-item modified Medical Outcomes Study Social Support Survey: Psychometric evaluation showed excellent performance. J Clin Epidemiol, 65, 1107-16.

Naoum FA (2016). Iron deficiency in cancer patients. Rev Bras Hematol Hemoter, 38, 325-30.

$\mathrm{Ng} \mathrm{CG}$, Mohamed S, See MH, et al (2015). Anxiety, depression, perceived social support and quality of life in Malaysian breast cancer patients: a 1-year prospective study. Health Qual Life Outcomes, 13, 205.

O'Brien PD, Hinder LM, Callaghan BC, Feldman EL (2017). Neurological consequences of obesity. Lancet Neurol, 16, 465-77.

Patel JD, Krilov L, Adams S, et al (2014). Clinical cancer advances 2013: Annual report on progress against cancer from the American Society of Clinical Oncology. J Clin Oncol, 32, 129-60.

Paxton RJ, Phillips KL, Jones LA, et al (2012). Associations among physical activity, body mass index, and health-related quality of life by race/ethnicity in a diverse sample of breast cancer survivors. Cancer, 118, 4024-31.

Quinten C, Coens C, Mauer M, et al (2009). Baseline quality of life as a prognostic indicator of survival: a meta-analysis of individual patient data from EORTC. Lancet Oncol, 10, 865-71.

Rahman MM, Ahsan MA, Monalisa NN, Rahman K (2014). Influence of socioeconomic status and BMI on the quality of life after mastectomy in Bangladeshi breast cancer patients in a public hospital. Jpn J Clin Oncol, 44, 1150-7.

Rohani C, Abedi H-A, Omranipour R, Langius-Eklöf A (2015). Health-related quality of life and the predictive role of sense of coherence, spirituality and religious coping in a sample of Iranian women with breast cancer: a prospective study with comparative design. Health Qual Life Outcomes, 13, 40.

Romero-Corral A, Caples SM, Lopez-Jimenez F, Somers VK (2010). Interactions between obesity and obstructive sleep apnea: Implications for treatment. Chest, 137, 711-9.

Scott NW, Fayers PM, Aaronson NK, et al (2008). EORTC QLQ-C30 Reference Values. EORTC Quality of Life Group PubLication, Brussels. Retrieved from https://www. eortc.org/app/uploads/sites/2/2018/02/reference_values_ manual2008.pdf.

Shi B, Chen J (2017). Handgrip strength may not accurately reflect the overall nutritional status of patients. Clin Nutr, 36, 316 .

Torre LA, Siegel RL, Ward EM, Jemal A (2015). Global cancer incidence and mortality rates and trends--An Update. Cancer Epidemiol Biomarkers Prev, 25, 16-27.

Van den Berg MMGA, Winkels RM, de Kruif JTCM, et al (2017). Weight change during chemotherapy in breast cancer 
patients: a meta-analysis. BMC Cancer, 17, 259.

Vance V, Mourtzakis M, Mccargar L, Hanning R (2011). Weight gain in breast cancer survivors: Prevalence, pattern and health consequences. Obesity Rev, 12, 282-94.

Von Hippel P, Benson R (2014). Obesity and the natural environment across US counties. Am J Public Health, 104, 1287-93.

Wayne SJ, Baumgartner K, Baumgartner RN, et al (2006). Diet quality is directly associated with quality of life in breast cancer survivors. Breast Cancer Res Treat, 96, 227-32.

World Health Organization. Regional Office for the Western Pacific (2000). The Asia-Pacific perspective: redefining obesity and its treatment. Sydney: Health Communications Australia.

Xia J, Tang Z, Deng Q, Wang J, Yu J (2018). Being slightly overweight is associated with a better quality of life in breast cancer survivors. Sci Rep, 8, 1-8.

Yu K, Zhou XR, He SL (2013). A multicentre study to implement nutritional risk screening and evaluate clinical outcome and quality of life in patients with cancer. Eur J Clin Nutr, 67, 732-7.

This work is licensed under a Creative Commons AttributionNon Commercial 4.0 International License. 